\title{
openheart Incidence of cardiovascular events and gastrointestinal bleeding in patients receiving clopidogrel with and without proton pump inhibitors: an updated meta-analysis
}

Rhanderson N Cardoso, ${ }^{1}$ Alexandre M Benjo, ${ }^{2}$ James J DiNicolantonio, ${ }^{3}$ Daniel C Garcia, ${ }^{1}$ Francisco Y B Macedo, ${ }^{4}$ Georges El-Hayek, ${ }^{5}$ Girish N Nadkarni, ${ }^{6}$ Sebastiano Gili, ${ }^{7}$ Mario lannaccone, ${ }^{7}$ Ioannis Konstantinidis, ${ }^{6}$ John P Reilly ${ }^{2}$

To cite: Cardoso RN, Benjo AM, DiNicolantonio JJ, et al. Incidence of cardiovascular events and gastrointestinal bleeding in patients receiving clopidogrel with and without proton pump inhibitors: an updated meta-analysis. Open Heart 2015;2:e000248.

doi:10.1136/openhrt-2015000248

\section{- Additional material is available. To view please visit the journal (http://dx.doi.org/ 10.1136/openhrt-2015- 000248). \\ RNC and AMB contributed equally and share first authorship.}

Received 7 February 2015 Revised 12 May 2015 Accepted 2 June 2015

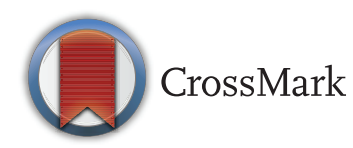

For numbered affiliations see end of article.

Correspondence to Dr Rhanderson N Cardoso; rhmncardoso@gmail.com

\section{ABSTRACT}

Background: Dual antiplatelet therapy is the standard of care after coronary stent placement but increases the bleeding risk. The effects of proton pump inhibitors (PPIs) on clopidogrel metabolism have been described, but the clinical significance is not yet definitive. We aimed to do an updated meta-analysis comparing outcomes in patients receiving clopidogrel with and without PPIs.

Methods: We systematically searched PubMed, Scopus and the Cochrane Central Register of Controlled Trials for randomised controlled trials (RCTs) and controlled observational studies in patients taking clopidogrel stratified by concomitant PPI use. Heterogeneity was examined with the Cochran $Q$ test and $\mathrm{I}^{2}$ statistics; $p$ values inferior to 0.10 and $\mathrm{I}^{2}>25 \%$ were considered significant for heterogeneity.

Results: We included 39 studies with a total of 214851 patients, of whom $73731(34.3 \%)$ received the combination of clopidogrel and a PPI. In pooled analysis, all-cause mortality, myocardial infarction, stent thrombosis and cerebrovascular accidents were more common in patients receiving both drugs. However, among 23552 patients from eight RCTs and propensity-matched studies, there were no significant differences in mortality or ischaemic events between groups. The use of PPIs in patients taking clopidogrel was associated with a significant reduction in the risk of gastrointestinal bleeding.

Conclusions: The results of our meta-analysis suggest that PPIs are a marker of increased cardiovascular risk in patients taking clopidogrel, rather than a direct cause of worse outcomes. The pharmacodynamic interaction between PPIs and clopidogrel most likely has no clinical significance. Furthermore, PPIs have the potential to decrease gastrointestinal bleeding in clopidogrel users.

\section{INTRODUCTION}

Dual antiplatelet therapy (DAPT) with aspirin and clopidogrel is recommended following

\section{KEY MESSAGES}

What is already known about this subject?

- Proton pump inhibitors have the potential to decrease the risk of upper gastrointestinal haemorrhage among patients taking antiplatelet therapy. However, pharmacokinetic data and observational studies have suggested a potential interaction between clopidogrel and proton pump inhibitors, which could have a significant effect in clinical events.

What does this study add?

- We studied potential factors associated with the interaction between clopidogrel and proton pump inhibitors, such as stent placement, presentation as an acute coronary syndrome, use of dual antiplatelet therapy, and stratification by different proton pump inhibitors. Importantly, in a subanalysis of studies with randomised or propensity score matched data, no significant difference was observed in adverse outcomes between patients who received a proton pump inhibitor and those who did not. The reduction in gastrointestinal bleeding among patients taking a proton pump inhibitor was consistent throughout the different subgroups.

How might this impact on clinical practice?

- The results of our study suggest that the previously reported interaction between clopidogrel and proton pump inhibitors may be dependent on selection bias and different patient baseline characteristics, as a clinically significant effect was not observed in a randomised/propensity score matched population. On the basis of these findings, physicians may consider proton pump inhibitors for patients receiving clopidogrel, as there is a benefit in terms of reduced gastrointestinal bleeding.

acute coronary syndromes (ACS) and percutaneous coronary interventions (PCI), as it 
has been shown to decrease the risk of adverse cardiovascular (CV) events. ${ }^{1-5}$ PPIs significantly decrease the risk of upper gastrointestinal (GI) haemorrhage in patients receiving antiplatelet therapy. ${ }^{6-8}$

Clopidogrel activation is dependent on the hepatic cytochrome $\mathrm{P} 450$, which can be competitively inhibited by PPIs. ${ }^{9-12}$ The potential interaction between clopidogrel and PPIs has been extensively demonstrated in pharmacokinetic platelet aggregation studies. ${ }^{13-16}$ These findings led to label warnings from the Food and Drug Administration regarding the concomitant use of clopidogrel with omeprazole or esomeprazole. ${ }^{17}$ Furthermore, these concerns have resulted in more restricted guideline indications for PPIs in patients taking antiplatelet therapy. ${ }^{18}$

Nevertheless, the majority of data on the clinical significance of the PPI-clopidogrel interaction derive from observational studies and the results have been conflicting. ${ }^{19-23}$ Two randomised controlled trials (RCTs) have failed to show an increased incidence of ischaemic CV outcomes in patients on concomitant use of clopidogrel and a PPI. ${ }^{7}{ }^{24}$ Multiple meta-analyses have been performed, but the most recent one included data only until June 2012. ${ }^{25-29}$ A substantial number of studies have been published since then, including over 50000 patients. ${ }^{30-36}$ We aimed to perform an updated meta-analysis comparing the incidence of adverse CV and GI events in patients receiving clopidogrel with and without PPIs. Furthermore, we sought to identify possible factors in the clopidogrel-PPI interaction, such as ACS, DAPT and specific PPIs.

\section{MATERIAL AND METHODS}

\section{Eligibility criteria and data extraction}

We restricted our analysis to studies that met all the following inclusion criteria: (1) RCTs, case-control or cohort (retrospective or prospective) studies; (2) patients on clopidogrel stratified into two groups: concomitant PPI-clopidogrel use versus clopidogrel use alone; (3) available data on any of the outcomes of interest in a direct comparison between PPI and non-PPI users; and (4) at least 6 months of follow-up. Exclusion criteria were non-controlled studies (absence of comparison group on clopidogrel without concomitant PPI use), ongoing studies and duplicate reports. In studies with outcomes reported in person-years rather than in absolute values, we attempted contact with the authors to obtain patientlevel data.

Each of the four authors (RNC, DCG, FYBM, GEH) independently extracted data following the defined search criteria and quality assessment. Disagreements between these four authors were resolved by consensus. In addition to outcomes of interest, the authors also extracted further information for subgroup analyses, including population characteristics, specific PPI used, concomitant use of aspirin and study design.

\section{Search strategy}

We systematically searched PubMed, Scopus and the Cochrane Central Register of Controlled Trials for RCTs and controlled observational studies in patients taking clopidogrel stratified by concomitant PPI use. The search was conducted without date restrictions in February 2014 for studies published in English only. The following medical subject heading terms were included: (clopidogrel OR Plavix) AND (PPI OR proton pump inhibitor OR omeprazole OR esomeprazole OR rabeprazole OR pantoprazole OR lansoprazole OR ilaprazole OR dexlansoprazole). In addition to searching databases, investigators also reviewed abstracts from the main cardiology and GI conferences from 2010 to 2014. Reference lists of all included studies, meta-analysis and reviews were manually searched. There was no patient population size restriction for the search.

\section{End points and subgroup analyses}

Outcomes of interest included all-cause mortality, CV mortality, myocardial infarction (MI), ACS, stent thrombosis, revascularisation, cerebrovascular accidents (CVA) and GI bleeding. Given the large number of studies and availability of individual outcomes, combined end points were not used. For the outcome of stent thrombosis, thought to be the most prone to variability in definitions, a subanalysis was performed including only definite cases according to Academic Research Consortium criteria. ${ }^{37}$ Owing to an anticipated variability in the definitions of GI bleeding, we restricted our analysis to gastric or duodenal bleeding confirmed by endoscopy.

In the search for potential factors associated with a clopidogrel-PPI interaction, prespecified subgroup analyses were performed. These included (1) concomitant treatment with aspirin (DAPT); (2) patients with PCI; (3) patients with ACS; and (4) stratification by risk of clopidogrel interaction according to degree of CYP450 2C19 inhibition. The high-risk PPI group included omeprazole, esomeprazole and lansoprazole, which are considered the most prone to CYP450 2C19 inhibition. ${ }^{11} 1617{ }^{20}$ Pantoprazole and rabeprazole were analysed separately in the low-risk PPI group. ${ }^{16} \quad 20 \quad 27 \quad 38$ Finally, a subanalysis was also performed that was restricted to RCTs and propensity score matched (PSM) studies to evaluate for the possibility of selection bias in observational studies.

\section{Quality assessment}

The quality of case-control and cohort studies was evaluated by the Newcastle-Ottawa Scale (NOS). ${ }^{39}$ This tool for quality assessment of non-randomised studies attributes none to nine stars according to the methodological quality of three parameters: selection of participants; comparability of groups; and assessment of either exposure in case-controls or outcomes in cohort studies. Previous meta-analyses have considered studies with 6 or more stars as high quality. ${ }^{40}{ }^{41}$ Post hoc analyses of RCTs were assessed as cohort studies by the 
NOS, given that the exposure of interest was not a randomised factor. With the exception of the two conference abstracts, all case-control and cohort studies included received a score of 7 or higher on the Newcastle-Ottawa scale and therefore were considered studies of high methodological quality. Quality assessment of RCTs was performed with the Jadad score, which evaluates randomisation, blinding and follow-up. ${ }^{42}$ Publication bias was evaluated by using funnel-plot graphs and checking for symmetrical distribution of trials with similar weights. ${ }^{43}$

\section{Statistical analysis}

Meta-analysis was performed according to recommendations of the Cochrane Collaboration and in line with the Preferred Reporting Items for Systematic Reviews and Meta-Analysis (PRISMA) statement. ${ }^{44}$ Pooled treatment effects were estimated using OR with 95\% CIs for binary end points. We used the random-effects DerSimonian and Laird model because of the anticipated wide variability between studies, particularly among observational data. Nevertheless, results were confirmed with the Mantel-Haenszel fixed-effect model to avoid small studies being overly weighted. Heterogeneity was examined with the Cochran $Q$ test and $\mathrm{I}^{2}$ statistics; $\mathrm{p}$ values inferior to 0.10 and $\mathrm{I}^{2}>25 \%$ were considered significant for heterogeneity. ${ }^{45}$ For statistical analysis, we used Review Manager 5.1 (Nordic Cochrane Centre, The Cochrane Collaboration, Copenhagen, Denmark).

\section{RESULTS}

\section{Study selection and characteristics}

As illustrated in figure 1, overall 2125 studies were identified. After removal of duplicate reports, animal studies and non-relevant studies by title or abstract review, 93 articles remained. These were fully reviewed for satisfaction of inclusion criteria. The main reasons for withdrawal were absence of control group, outcomes of interest not reported or a short follow-up interval.

Thirty-seven manuscripts met all criteria and were included. An additional two studies were included from a review of conference abstracts. A total of 39 studies and 214851 patients were included, of whom 73731 $(34.3 \%)$ received the combination of clopidogrel and a PPI. The vast majority were observational studies and only three RCTs were identified. Study characteristics are presented in table 1. Baseline characteristics in individual studies were most commonly not comparable between groups; therefore, a subanalysis of RCTs/propensity score matched (PSM) populations was carried out to evaluate the impact of selection bias in study results.

\section{Pooled analysis of all studies}

All-cause mortality (OR 1.39; 95\% CI 1.19 to 1.61 ; $\mathrm{p}<0.001)$ and MI (OR 1.41; 95\% CI 1.20 to 1.65 ; $\mathrm{p}<0.001)$ were significantly increased in the group of patients receiving PPIs, as illustrated in figure 2. Stent thrombosis (OR 1.30; 95\% CI 1.05 to 1.63 ; $\mathrm{p}=0.02$ ), definite stent thrombosis (OR 1.65; 95\% CI 1.10 to 2.48; $\mathrm{p}=0.02$; figure $3 \mathrm{~A}$ ), ACS (OR 1.92; 95\% CI 1.23 to 3.0; $\mathrm{p}=0.004$; figure $3 \mathrm{~B}$ ) and CVA (OR 1.66; $95 \%$ CI 1.40 to $1.97 ; \mathrm{p}<0.001$; figure $3 \mathrm{C}$ ) were also more common in patients receiving both drugs. There was also a strong trend towards increased revascularisation (OR 1.26; 95\% CI 1.0 to $1.59 ; \mathrm{p}=0.05$; figure $3 \mathrm{D}$ ) in the PPI-clopidogrel group. Conversely, the concomitant use of PPIs had a protective effect over the risk of GI bleeding (OR 0.40;
Figure 1 Preferred Reporting Items for Systematic Reviews and Meta-Analysis (PRISMA) flow diagram of study selection.

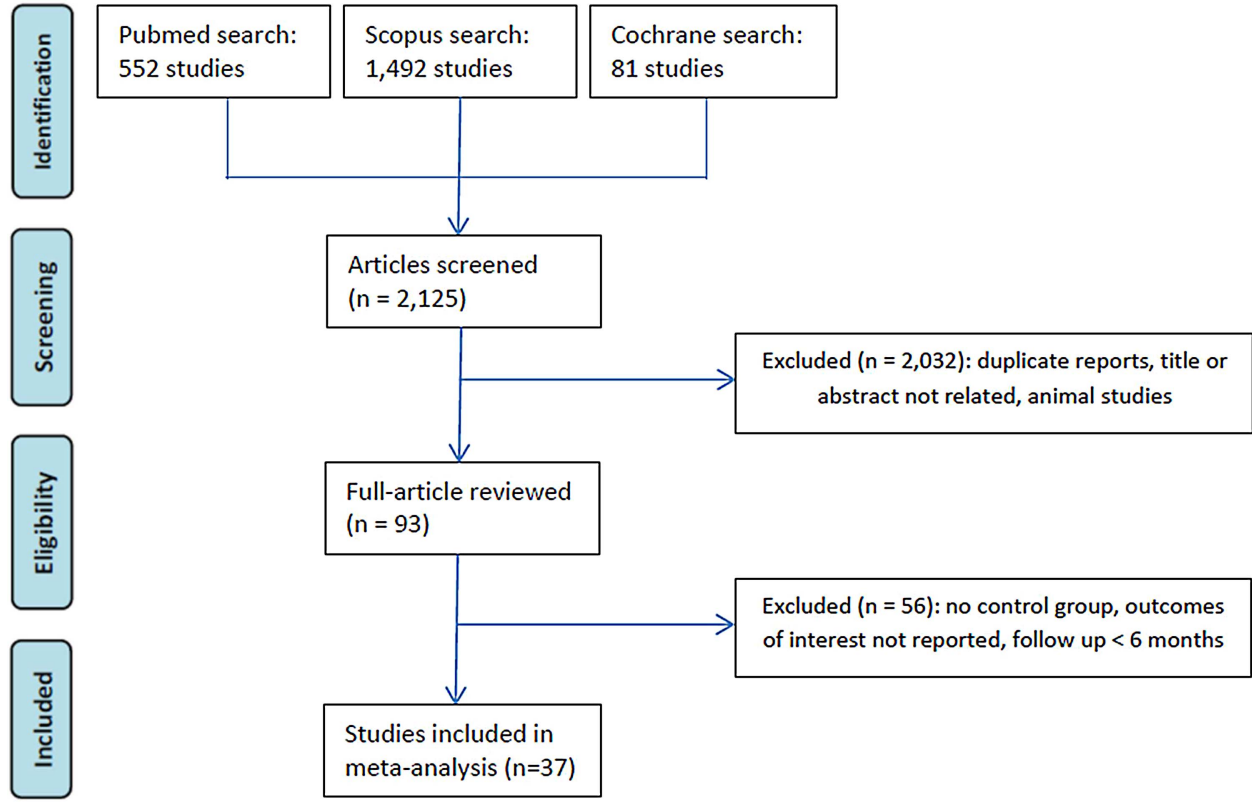


Table 1 Characteristics of studies included in meta-analysis

\begin{tabular}{|c|c|c|c|c|c|c|c|c|c|}
\hline $\begin{array}{l}\text { Study } \\
\text { Design }\end{array}$ & Study & Patients (n) & Location & Time frame & Follow-up & PPIs & Patient population & Outcomes available & DAT \\
\hline Case-control & Juurlinket $a^{46}$ & $\begin{array}{l}\text { PPI: } 170 \\
\text { No PPI: } 407\end{array}$ & Ontario, Canada & $\begin{array}{l}4 / 2002-9 / \\
2008\end{array}$ & 6 months & $L, O, P, R$ & CVA & All-cause mortality, CVA & $<9 \%$ \\
\hline Case-control & Valkhoffet $a f^{47}$ & $\begin{array}{l}\text { PPI: } 4793 \\
\text { No } \\
\text { PPI:11 } 237\end{array}$ & Netherlands & $\begin{array}{l}1 / 1999- \\
12 / 2008\end{array}$ & 42.6 months & $\begin{array}{l}\mathrm{E}, \mathrm{L}, \mathrm{O} \\
\mathrm{P}, \mathrm{R}\end{array}$ & MI & MI & NA \\
\hline Case-control & Ching et $a f^{48}$ & $\begin{array}{l}\text { PPI: } 1128 \\
\text { No PPI: } 2159\end{array}$ & Connecticut, USA & $\begin{array}{l}1 / 2004- \\
11 / 2008\end{array}$ & 9 months & $\begin{array}{l}\text { E, L, O, } \\
P, R\end{array}$ & $\mathrm{PCl}$ with stent & All-cause mortality, MI, revascularisation (TVR) & Yes \\
\hline Case-control & Jiang et $a \beta^{34}$ & $\begin{array}{l}\text { PPI: } 1570 \\
\text { No PPI: } 1110\end{array}$ & Nanjing, China & $\begin{array}{l}1 / 2008- \\
1 / 2011\end{array}$ & 1 year & $E, L, O$ & $\mathrm{PCl}$ & GI bleed & Yes \\
\hline Case-control & $\begin{array}{l}\text { Garcia Rodriguez } \\
\text { et } a^{\beta 2}\end{array}$ & $\begin{array}{l}\text { PPI: } 192 \\
\text { No PPI: } 177\end{array}$ & UK & $\begin{array}{l}1 / 2000- \\
12 / 2007\end{array}$ & $>3.5$ years & $\begin{array}{l}\text { E, L, O, } \\
P, R\end{array}$ & ACS & Gl bleed & NA \\
\hline Retrospective cohort & Ho et $a^{49}$ & $\begin{array}{l}\text { PPI: } 5244 \\
\text { No PPI: } 2961\end{array}$ & VA hospitals, USA & $\begin{array}{l}8 / 2003- \\
1 / 2006\end{array}$ & 521 days & $\mathrm{L}, \mathrm{O}, \mathrm{P}, \mathrm{R}$ & ACS & All-cause mortality, ACS, revascularisation & $\sim 90 \%$ \\
\hline Retrospective cohort & Evanchan et $a^{50}$ & $\begin{array}{l}\text { PPI: } 1369 \\
\text { No PPI: } 4425\end{array}$ & Ohio, USA & $\begin{array}{l}1 / 2003- \\
1 / 2008\end{array}$ & 1 year & $E, L, O, P$ & Stent after MI & MI & NA \\
\hline Retrospective cohort & Gaglia et $a^{p^{1}}$ & $\begin{array}{l}\text { PPI: } 318 \\
\text { No PPI: } 502\end{array}$ & Washington DC, USA & $\begin{array}{l}4 / 2003- \\
4 / 2007\end{array}$ & 1 year & $\begin{array}{l}E, L, O \\
P, R\end{array}$ & $\mathrm{PCl}$ with DES & All-cause mortality, MI, revascularisation (TVR), ST & Yes \\
\hline Retrospective cohort & Gaspar et $a^{{ }^{2}}$ & $\begin{array}{l}\text { PPI: } 274 \\
\text { No PPI: } 528\end{array}$ & Braga, Portugal & $\begin{array}{l}1 / 2004- \\
3 / 2008\end{array}$ & 6 months & L, O, R & ACS & All-cause mortality, ACS & Yes \\
\hline Retrospective cohort & Gupta et $a^{F^{3}}$ & $\begin{array}{l}\text { PPI: } 72 \\
\text { No PPI: } 243\end{array}$ & Little Rock, AR, USA & $\begin{array}{l}1 / 2003- \\
8 / 2004\end{array}$ & 4 years & L, O, R & $\mathrm{PCl}$ with stent & All-cause mortality, revascularisation (TLR) & $>95 \%$ \\
\hline Retrospective cohort & Kreutz et $a^{F^{4}}$ & $\begin{array}{l}\text { PPI: } 6828 \\
\text { No PPI: } 9862\end{array}$ & $\begin{array}{l}\text { Medco Health Database, } \\
\text { USA }\end{array}$ & $\begin{array}{l}\text { 10/2005- } \\
9 / 2006\end{array}$ & 1 year & $\begin{array}{l}\text { E, L, O, } \\
P, R\end{array}$ & $\mathrm{PCl}$ with stent & CV death, MI, ACS, revascularisation, CVA & NA \\
\hline Retrospective cohort & Tentzeris et $a^{{ }^{5}}$ & $\begin{array}{l}\text { PPI: } 691 \\
\text { No PPI: } 519\end{array}$ & Vienna, Austria & $\begin{array}{l}1 / 2003- \\
12 / 2006\end{array}$ & 7.8 months & $\begin{array}{l}\text { E, L, O, } \\
P, R\end{array}$ & $\mathrm{PCl}$ with stent & All-cause mortality, CV mortality, ACS, ST & Yes \\
\hline Retrospective cohort & van Boxel et $a f^{3}$ & $\begin{array}{l}\text { PPI: } 5734 \\
\text { No } \\
\text { PPI:12 } 405\end{array}$ & The Netherlands & $\begin{array}{l}1 / 2006- \\
12 / 2007\end{array}$ & $1-2$ years & $\begin{array}{l}\text { E, L, O, } \\
P, R\end{array}$ & NA & All-cause mortality, MI, ACS, CVA & $\sim 78 \%$ \\
\hline Retrospective cohort & Wu et $a^{56}$ & $\begin{array}{l}\text { PPI: } 311 \\
\text { No PPI: } 5551\end{array}$ & Taiwan & $\begin{array}{l}7 / 2002- \\
6 / 2005\end{array}$ & 1 year & $\begin{array}{l}E, L, O \\
P, R\end{array}$ & ACS & ACS, revascularisation & NA \\
\hline Retrospective cohort & Yasu et $a^{57}$ & $\begin{array}{l}\text { PPI: } 103 \\
\text { No PPI: } 188\end{array}$ & Kamakura, Japan & $\begin{array}{l}6 / 2006- \\
3 / 2009\end{array}$ & 395 days & $\mathrm{R}$ & $\mathrm{PCl}$ with DES & CV mortality, ACS, revascularisation, ST, GI bleed & Yes \\
\hline Retrospective cohort & Munoz-Torrero et $a^{58}$ & $\begin{array}{l}\text { PPI: } 519 \\
\text { No PPI: } 703\end{array}$ & Spain & $\begin{array}{l}3 / 2003- \\
3 / 2009\end{array}$ & 15 months & $\mathrm{L}, \mathrm{O}, \mathrm{P}$ & $\begin{array}{l}\text { Atherosclerotic } \\
\text { disease }\end{array}$ & All-cause mortality, MI, CVA & $\sim 65 \%$ \\
\hline Retrospective cohort & Hauptle et $a l^{\beta 3}$ & $\begin{array}{l}\text { PPI: } 87 \\
\text { No PPI: } 631\end{array}$ & Switzerland & $\begin{array}{l}1 / 2005- \\
12 / 2006\end{array}$ & 1 year & $\begin{array}{l}\text { E, L, O, } \\
P, R\end{array}$ & $\mathrm{PCl}$ for ACS & Gl bleed & Yes \\
\hline Retrospective cohort & Ortolani et $a^{59}$ & $\begin{array}{l}\text { PPI: } 3519 \\
\text { No PPI: } 377\end{array}$ & Emilia-Romagna, Italy & $\begin{array}{l}1 / 2008- \\
8 / 2008\end{array}$ & 1 year & $\begin{array}{l}\text { E, L, O, } \\
P, R\end{array}$ & ACS & All-cause mortality, ACS, revascularisation & $>91 \%$ \\
\hline Retrospective cohort & Nakayama et a ${ }^{\beta 5}$ & $\begin{array}{l}\text { PPI: } 280 \\
\text { No PPI: } 284\end{array}$ & Tokyo, Japan & $\begin{array}{l}1 / 2005- \\
12 / 2009\end{array}$ & 880 days & L, O, R & $\mathrm{PCl}$ & Revascularisation (TLR) & Yes \\
\hline Retrospective cohort & Zou et $a \beta^{\beta 6}$ & $\begin{array}{l}\text { PPI: } 6188 \\
\text { No PPI: } 1465\end{array}$ & Nanjing, China & $\begin{array}{l}10 / 2005- \\
9 / 2010\end{array}$ & 1 year & $E, O, P$ & ACS with DES & $\mathrm{CV}$ death, MI, revascularisation (TVR), ST & Yes \\
\hline Cohort & Zairis et $a^{60}$ & $\begin{array}{l}\text { PPI: } 340 \\
\text { No PPI: } 248\end{array}$ & Piraeus, Greece & $\begin{array}{l}4 / 2003- \\
1 / 2005\end{array}$ & 1 year & 0 & $\mathrm{PCl}$ with stent & CV mortality, MI, revascularisation, ST & $>97 \%$ \\
\hline Cohort & $\begin{array}{l}\text { Hokimoto and } \\
\text { Ogawa }^{61}\end{array}$ & $\begin{array}{l}\text { PPI: } 37 \\
\text { No PPI: } 133\end{array}$ & Japan & NA & 1 year & $\mathrm{R}$ & DAT & $\mathrm{CV}$ mortality, ACS, revascularisation, CVA, GI bleed & Yes \\
\hline Cohort & Hudzik et a ${ }^{2}$ & $\begin{array}{l}\text { PPI: } 18 \\
\text { No PPI: } 20\end{array}$ & Poland & $\begin{array}{l}1 / 2006- \\
7 / 2008\end{array}$ & 1 year & $\mathrm{O}$ & $\mathrm{PCl}$ with stent & MI, ACS, CVA & Yes \\
\hline Cohort & Banerjee et $a^{63}$ & $\begin{array}{l}\text { PPI: } 867 \\
\text { No PPI: } 3678\end{array}$ & $\begin{array}{l}\text { Veteran Affairs Database, } \\
\text { USA }\end{array}$ & $\begin{array}{l}1 / 2003- \\
12 / 2008\end{array}$ & 1 year & $\begin{array}{l}E, L, O \\
P, R\end{array}$ & $\mathrm{PCl}$ with stent & All-cause mortality, MI, revascularisation & NA \\
\hline
\end{tabular}




\begin{tabular}{|c|c|c|c|c|c|c|c|c|c|}
\hline $\begin{array}{l}\text { Study } \\
\text { Design }\end{array}$ & Study & Patients (n) & Location & Time frame & Follow-up & PPIs & Patient population & Outcomes available & DAT \\
\hline Cohort & Rossini et $a^{\beta 4}$ & $\begin{array}{l}\text { PPI: } 1158 \\
\text { No PPI: } 170\end{array}$ & Northern Italy & NA & 1 year & $\mathrm{L}, \mathrm{O}, \mathrm{P}$ & $\mathrm{PCl}$ with DES & All-cause mortality, ST & Yes \\
\hline Cohort & Simon et $a^{P^{2}}$ & $\begin{array}{l}\text { PPI: } 1052 \\
\text { No PPI: } 711\end{array}$ & France & NA & 1 year & $E, L, O, P$ & MI & All-cause mortality & NA \\
\hline Cohort & Chitose et $a{ }^{65}$ & $\begin{array}{l}\text { PPI: } 187 \\
\text { No PPI: } 443\end{array}$ & Multicenter, Japan & $\begin{array}{l}6 / 2008- \\
3 / 2009\end{array}$ & 18 months & L, O, R & $\mathrm{PCl}$ with stent & CV mortality, MI, CVA & Yes \\
\hline Cohort & Douglas et $a \beta^{\beta 1}$ & $\begin{array}{l}\text { PPI: } 12439 \\
\text { No } \\
\text { PPI:16 } 900\end{array}$ & UK & $\begin{array}{l}1 / 2003- \\
7 / 2009\end{array}$ & 303 days & $E, L, O$ & UK national database & All-cause mortality, CV mortality, MI & Yes \\
\hline $\begin{array}{l}\text { Post hoc analysis of } \\
\text { RCT }\end{array}$ & O'Donoghue et $a^{\beta 6}$ & $\begin{array}{l}\text { PPI: } 2257 \\
\text { No PPI: } 4538\end{array}$ & Multinational & $<9 / 2007$ & 15 months & $\begin{array}{l}E, L, O \\
P, R\end{array}$ & $\begin{array}{l}\mathrm{PCl} \text { for } \mathrm{ACS},>94 \% \\
\text { stent }\end{array}$ & All-cause mortality, CV mortality, MI, ST & $>96 \%$ \\
\hline $\begin{array}{l}\text { Post hoc analysis of } \\
\text { RCT }\end{array}$ & Burkard et $a^{67}$ & $\begin{array}{l}\text { PPI: } 109 \\
\text { No PPI: } 692\end{array}$ & Basel, Switzerland & $\begin{array}{l}5 / 2003- \\
5 / 2004\end{array}$ & 36 months & $E, L, O, P$ & $\mathrm{PCl}$ with stent & $\begin{array}{l}\text { All-cause mortality, CV mortality, MI, } \\
\text { revascularisation (TVR), ST }\end{array}$ & $>91 \%$ \\
\hline $\begin{array}{l}\text { Post hoc analysis of } \\
\text { RCT }\end{array}$ & Goodman et $a^{68}$ & $\begin{array}{l}\text { PPI: } 3255 \\
\text { No PPI: } 6021\end{array}$ & Multinational & $\begin{array}{l}10 / 2006- \\
7 / 2008\end{array}$ & 1 year & $\begin{array}{l}E, L, O \\
P, R\end{array}$ & ACS & All-cause mortality, CV mortality, ST & $>97 \%$ \\
\hline PSM cohort & Harjai et $a l^{19}$ & $\begin{array}{l}\text { PPI: } 685 \\
\text { No PPI: } 685\end{array}$ & Sayre, PA, USA & $\begin{array}{l}7 / 2001- \\
12 / 2007\end{array}$ & 6 months & $\mathrm{E}, \mathrm{O}$ & $\mathrm{PCl}$ & All-cause mortality, MI, revascularisation (TVR), ST & $>98 \%$ \\
\hline PSM cohort & Hsiao et $a^{69}$ & $\begin{array}{l}\text { PPI: } 622 \\
\text { No PPI: } 9131\end{array}$ & Taiwan & $2000-2007$ & $>6$ months & $\begin{array}{l}E, L, O \\
P, R\end{array}$ & ACS & ACS & Yes \\
\hline PSM cohort & Aihara et $a l^{70}$ & $\begin{array}{l}\text { PPI: } 1068 \\
\text { No PPI: } 819\end{array}$ & Ibakari, Japan & $\begin{array}{l}2 / 2006- \\
8 / 2009\end{array}$ & 1 year & L, O, R & $\mathrm{PCl}$ with stent & $\begin{array}{l}\text { All-cause mortality, MI, revascularisation, ST, CVA, } \\
\text { Gl bleed }\end{array}$ & Yes \\
\hline PSM cohort & Bhurke et $a^{\beta 0}$ & $\begin{array}{l}\text { PPI: } 2958 \\
\text { No PPI: } 7143\end{array}$ & USA & $\begin{array}{l}1 / 2001- \\
12 / 2008\end{array}$ & 268 days & $\begin{array}{l}E, L, O \\
P, R\end{array}$ & ACS & $\mathrm{Ml}$, revascularisation & NA \\
\hline PSM cohort & Lin et $a l^{71}$ & $\begin{array}{l}\text { PPI: } 5173 \\
\text { No } \\
\text { PPI:31 } 926\end{array}$ & Taiwan & $\begin{array}{l}1 / 2006- \\
12 / 007\end{array}$ & 580 days & $\begin{array}{l}E, L, O \\
P, R\end{array}$ & ACS & ACS, revascularisation & NA \\
\hline RCT & Bhatt et al ${ }^{7}$ & $\begin{array}{l}\text { PPI: } 1876 \\
\text { No PPI: } 1885\end{array}$ & Multinational & $\begin{array}{l}1 / 2008- \\
12 / 2008\end{array}$ & 180 days & $\mathrm{O}$ & ACS or stent & $\begin{array}{l}\text { All-cause mortality, CV mortality, MI, } \\
\text { revascularisation, CVA, GI bleed }\end{array}$ & Yes \\
\hline $\mathrm{RCT}$ & Hsu et $a R^{4}$ & $\begin{array}{l}\text { PPI: } 83 \\
\text { No PPI: } 82\end{array}$ & Taiwan & $\begin{array}{l}8 / 2008- \\
1 / 2010\end{array}$ & 6 months & $\mathrm{E}$ & History of GI ulcer & MI, ACS, CVA & NA \\
\hline $\mathrm{RCT}$ & $\mathrm{Hsu}^{72}$ & $\begin{array}{l}\text { PPI: } 157 \\
\text { No PPI: } 161\end{array}$ & Taiwan & $\begin{array}{l}1 / 2008- \\
11 / 2010\end{array}$ & 6 months & $\mathrm{E}$ & History of GI ulcer & Gl bleed & NA \\
\hline
\end{tabular}

ACS: acute coronary syndrome; CV: cardiovascular; CVA: cerebrovascular accident; DAT: dual antiplatelet therapy; DES: drug-eluting stent E: esomeprazole; GI: gastrointestinal; L: lansoprazole; MI: myocardial infarction; NA: not available or not applicable; O: omeprazole; P: pantoprazole; PCI: percutaneous coronary intervention; PPI: proton pump inhibitor; PSM: propensity score matched; R: rabeprazole; RCT: randomised controlled trial; ST: stent thrombosis; TLR: target lesion revascularisation; TVR: target vessel revascularisation. 
A Overall mortality

\begin{tabular}{|c|c|c|c|c|c|c|c|c|c|}
\hline \multirow[b]{2}{*}{ Study or Subgroup } & \multicolumn{2}{|c|}{ PPI } & \multicolumn{2}{|c|}{ No PPI } & \multirow[b]{2}{*}{ Weight } & \multirow{2}{*}{$\begin{array}{c}\text { Odds Ratio } \\
\text { M.H. Random, 95\% CI }\end{array}$} & \multirow{2}{*}{\multicolumn{3}{|c|}{$\begin{array}{c}\text { Odds Ratio } \\
M \cdot \mathrm{H}, \text { Random, } 95 \% \mathrm{Cl}\end{array}$}} \\
\hline & Events & Total & Events & Total & & & & & \\
\hline Aihara 2012 & 36 & 1068 & 28 & 819 & $4.6 \%$ & $0.99[0.60,1.63]$ & & & 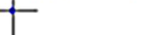 \\
\hline Banerjee 2011 & 232 & 867 & 787 & 3678 & $8.5 \%$ & $1.34[1.13,1.59]$ & & & - \\
\hline Bhatt 2010 COGENT & 5 & 1876 & 5 & 1885 & $1.3 \%$ & $1.00[0.29,3.48]$ & & & \\
\hline Burkard 2012 BASKET & 10 & 109 & 51 & 692 & $3.1 \%$ & $1.27[0.62,2.58]$ & & & \\
\hline Ching 2012 & 34 & 1128 & 24 & 2159 & $4.4 \%$ & $2.76[1,63,4.69]$ & & & $\rightarrow$ \\
\hline Douglas 2012 & 1170 & 12439 & 1058 & 16900 & $9.3 \%$ & $1.55[1.43,1.70]$ & & & - \\
\hline Gaglia 2010 & 15 & 318 & 9 & 502 & $2.4 \%$ & $2.71[1.17,6.27]$ & & & \\
\hline Gaspar 2010 & 18 & 274 & 21 & 528 & $3.5 \%$ & $1.70[0.89,3.24]$ & & & - \\
\hline Goodman 2012 PLATO & 213 & 3255 & 286 & 6021 & $8.4 \%$ & $1.40[1.17,1.69]$ & & & - \\
\hline Gupta 2010 & 14 & 72 & 35 & 243 & $3.2 \%$ & $1.43[0.72,2.84]$ & & & 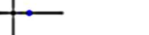 \\
\hline Harjai 2011 & 17 & 685 & 20 & 685 & $3.4 \%$ & $0.85[0.44,1.63]$ & & & - \\
\hline Ho 2009 & 1042 & 5244 & 493 & 2961 & $9.0 \%$ & $1.24[1.10,1.40]$ & & & - \\
\hline Juurlink 2011 & 27 & 101 & 35 & 205 & $4.0 \%$ & $1.77[1.00,3.14]$ & & & - \\
\hline Munoz-Torrero 2011 & 39 & 519 & 23 & 703 & $4.4 \%$ & $2.40[1.42,4.07]$ & & & $\rightarrow$ \\
\hline O'Donoghue 2009 TIMI-38 & 58 & 2257 & 139 & 4538 & $6.8 \%$ & $0.83[0.61,1.14]$ & & & \\
\hline Ortolani 2012 & 190 & 3519 & 16 & 377 & $4.5 \%$ & $1.29[0.76,2.17]$ & & & - \\
\hline Rossini 2011 & 24 & 1158 & 5 & 170 & $1.9 \%$ & $0.70[0.26,1.86]$ & & & - \\
\hline Simon 2011 & 94 & 1052 & 77 & 711 & $6.7 \%$ & $0.81[0.59,1.11]$ & & & \\
\hline Tentzeris 2010 & 15 & 691 & 11 & 519 & $2.7 \%$ & $1.02[0.47,2.25]$ & & & \\
\hline van Boxel 2010 & 189 & 5734 & 164 & 12405 & $8.0 \%$ & $2.54[2.06,3.14]$ & & & - \\
\hline Total $(95 \% \mathrm{CI})$ & & 42366 & & 56701 & $100.0 \%$ & $1.39[1.19,1.61]$ & & & $\downarrow$ \\
\hline Total events & 3442 & & 3287 & & & & & & \\
\hline $\begin{array}{l}\text { Heterogeneity. } \operatorname{Tau}^{2}=0.0 \\
\text { Test for overall effect: } Z=\end{array}$ & $\begin{array}{l}i^{2}=82 . \\
(P<0.0\end{array}$ & $\begin{array}{l}\text { 75. } d f= \\
001)\end{array}$ & $(P<0$. & 00001 & $=77 \%$ & & 0.0 & $\begin{array}{l}0.1 \\
\text { Favors PPI }\end{array}$ & Favors no \\
\hline
\end{tabular}

B Myocardial infarction

\begin{tabular}{|c|c|c|c|c|c|c|c|c|}
\hline Study or Subgroup & \multicolumn{2}{|c|}{ PPI } & \multicolumn{2}{|c|}{ No PPI } & Weight & $\begin{array}{l}\text { Odds Ratio } \\
\text { Random } 95\end{array}$ & \multicolumn{2}{|c|}{$\begin{array}{c}\text { Odds Ratio } \\
M \cdot \mathrm{H}, \text { Random, } 95 \% \mathrm{Cl}\end{array}$} \\
\hline Aihara 2012 & 9 & 1068 & 8 & 819 & $2.1 \%$ & $0.86[0.33,2.24]$ & & - \\
\hline Banerjee 2011 & 187 & 867 & 846 & 3678 & $8.2 \%$ & $0.92[0.77,1.10]$ & & \\
\hline Bhatt 2010 COGENT & 14 & 1876 & 15 & 1885 & $3.1 \%$ & $0.94[0.45,1.95]$ & & \\
\hline Bhurke 2012 & 196 & 2958 & 459 & 7143 & $8.3 \%$ & $1.03[0.87,1.23]$ & & \\
\hline Burkard 2012 BASKET & 16 & 109 & 51 & 692 & $3.9 \%$ & $2.16[1.18,3.95]$ & & \\
\hline Ching 2012 & 12 & 1128 & 13 & 2159 & $2.8 \%$ & $1.78[0.81,3.90]$ & & \\
\hline Chitose 2012 & 1 & 187 & 3 & 443 & $0.5 \%$ & $0.79[0.08,7.63]$ & & \\
\hline Douglas 2012 & 365 & 12345 & 369 & 16680 & $8.5 \%$ & $1.35[1.16,1.56]$ & & - \\
\hline Evanchan 2010 & 356 & 1369 & 693 & 4425 & $8.5 \%$ & $1.89[1.64,2.19]$ & & - \\
\hline Gaglia 2010 & 0 & 318 & 1 & 502 & $0.2 \%$ & $0.52[0.02,12.92]$ & & \\
\hline Goodman 2012 PLATO & 245 & 3255 & 354 & 6021 & $8.3 \%$ & $1.30[1.10,1.54]$ & & - \\
\hline Harjai 2011 & 20 & 667 & 20 & 665 & $3.7 \%$ & $1.00[0.53,1.87]$ & & \\
\hline Hsu 2011 & 2 & 83 & 2 & 82 & $0.6 \%$ & $0.99[0.14 .7 .18]$ & & \\
\hline Hudzik 2010 & 6 & 18 & 1 & 20 & $0.5 \%$ & $9.50[1.01,88.97]$ & & \\
\hline Kreutz 2010 & 475 & 6828 & 474 & 9862 & $8.6 \%$ & $1.48[1.30,1.69]$ & & - \\
\hline Munoz-Torrero 2011 & 29 & 519 & 15 & 703 & $3.7 \%$ & $2.71[1.44 .5 .12]$ & & \\
\hline ODonoghue 2009 TIMI-38 & 209 & 2257 & 424 & 4538 & $8.3 \%$ & $0.99[0.83,1.18]$ & & \\
\hline Valkhoff 2011 & 78 & 4793 & 90 & 11237 & $6.8 \%$ & $2.05[1.51,2.78]$ & & $\rightarrow$ \\
\hline van Boxel 2010 & 84 & 5734 & 78 & 12405 & $6.8 \%$ & $2.35[1.72,3.20]$ & & $\rightarrow$ \\
\hline Zairis 2010 & 22 & 340 & 16 & 248 & $3.5 \%$ & $1.00[0.52,1.95]$ & & 一 \\
\hline Zou 2014 & 69 & 6188 & 9 & 1465 & $3.3 \%$ & $1.82[0.91,3.66]$ & & \\
\hline Total $(95 \% \mathrm{Cl})$ & & 52907 & & 85672 & $100.0 \%$ & $1.41[1.20,1.65]$ & & $\diamond$ \\
\hline Total events & 2395 & & 3941 & & & & & \\
\hline $\begin{array}{l}\text { Heterogeneity: } \operatorname{Tau}^{2}=0.07 \\
\text { Test for overall effect: } Z=4\end{array}$ & $\begin{array}{l}\mathrm{hi}^{2}=96 . \\
(\mathrm{P}<0.0\end{array}$ & 6. $d f=$ & $20(P<0$. & 001 & $=79 \%$ & & $\begin{array}{lc}\stackrel{1}{0.01} & 0.1 \\
& \text { Favor }\end{array}$ & $\begin{array}{r}10 \\
\text { Favors no }\end{array}$ \\
\hline
\end{tabular}

Figure 2 Forest plot of studies examining outcomes between patients taking proton pump inhibitor (PPIs) with clopidogrel and those taking only clopidogrel: (A) overall mortality; (B) myocardial infarction.

$95 \%$ CI 0.22 to $0.74 ; \mathrm{p}=0.003$; figure 4$)$. A separate analysis restricted to cohort studies revealed similar results to the overall analysis.

\section{RCTs and propensity score matched studies}

Given the overwhelming majority of non-randomised observational studies in our meta-analysis and the subsequent risk of baseline heterogeneity between groups, a subanalysis was performed including only RCTs and
PSM populations. A total of 23552 patients were entered in the analysis, of whom 11770 (49.9\%) received the combination of clopidogrel and a PPI. Results are illustrated in figure 5 and show that all-cause mortality (OR $0.91 ; 95 \%$ CI 0.58 to $1.40 ; \mathrm{p}=0.66$ ), ACS (OR 0.96 ; $95 \%$ CI 0.88 to 1.05 ; $\mathrm{p}=0.35$ ), MI (OR 1.05; 95\% CI 0.86 to $1.28 ; \mathrm{p}=0.65$ ) and CVA (OR 1.47 ; $95 \%$ CI 0.66 to $3.25 ; \mathrm{p}=0.34$ ) were not significantly different between treatment groups. Revascularisation (OR 0.88; 

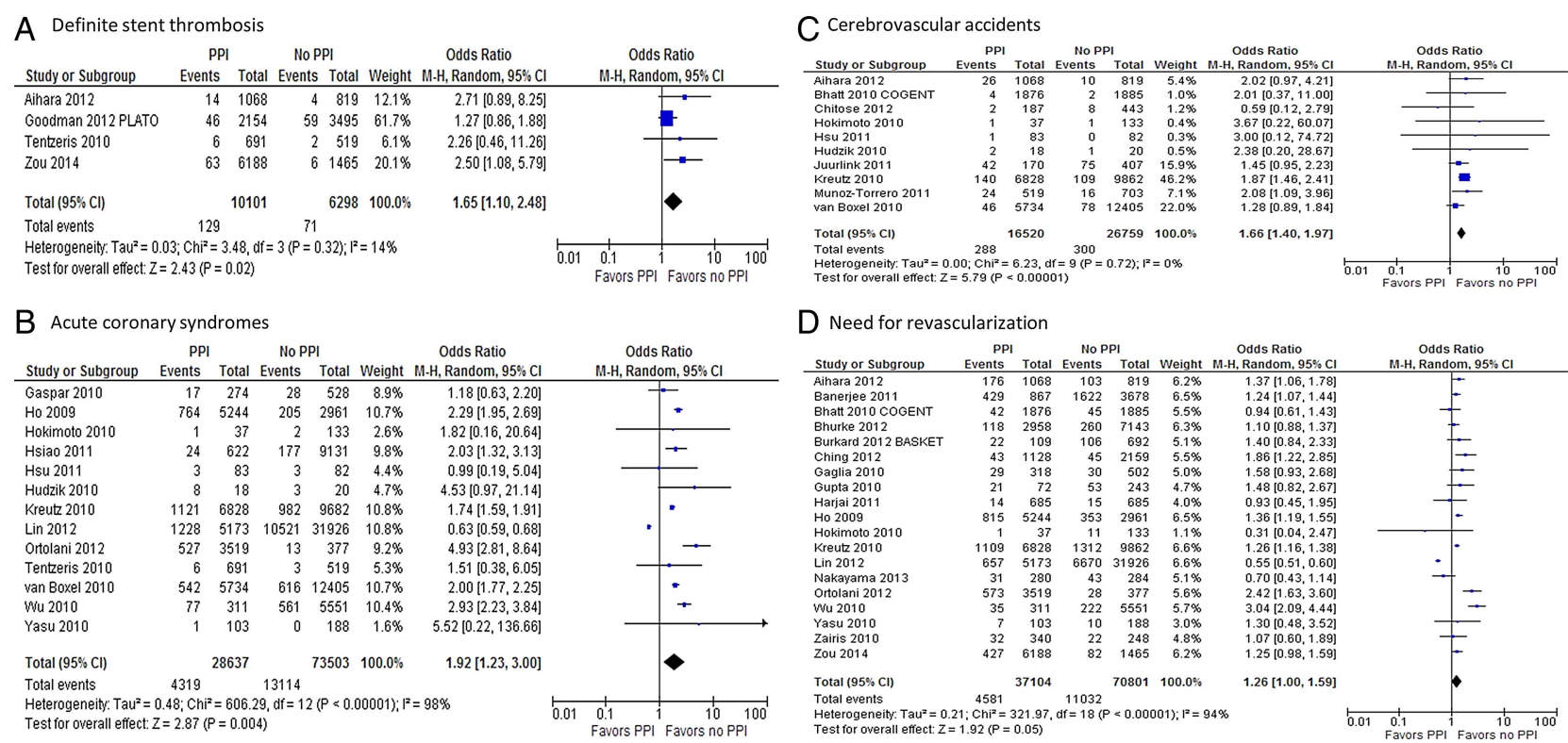

Figure 3 Forest plot of studies examining outcomes between patients taking proton pump inhibitor (PPIs) with clopidogrel and those taking only clopidogrel: (A) definite stent thrombosis; (B) need for revascularisation; (C) cerebrovascular accidents; (D) acute coronary syndromes.

95\% CI 0.80 to $0.97 ; \mathrm{p}=0.01$ ) was also not increased in patients who received concomitant PPI with clopidogrel. Furthermore, occurrence of GI bleed was significantly decreased in the group of patients who received a PPI (OR $0.24 ; 95 \%$ CI 0.09 to $0.62 ; \mathrm{p}=0.003$ ).

\section{Subgroup analyses}

Table 2 illustrates results of comparisons in studies with restricted populations. In studies limited to patients with ACS, only MI (OR 1.41; p=0.01) was significantly increased in patients taking clopidogrel with a concomitant PPI. In patients receiving DAPT, adding a PPI decreased the risk of an upper GI bleed (OR 0.31; $\mathrm{p}=0.002$ ), but was associated with increased risk of allcause mortality (OR 1.32; p=0.003), ACS (OR 2.37; $\mathrm{p}=0.002)$, MI (OR 1.25; $\mathrm{p}=0.005)$, stent thrombosis (OR 1.36; $\mathrm{p}=0.005$ ) and revascularisation (OR 1.30; $\mathrm{p}=0.006$ ). Stratification by degree of CYP450 2 C19 inhibition revealed that both high-risk (omeprazole, esomeprazole and lansoprazole) and low-risk PPIs (pantoprazole and rabeprazole) were associated with an increased risk of MI and mortality. In patients receiving high-risk PPIs, GI bleed was also decreased by concomitant PPI use (OR $0.17 ; \mathrm{p}<0.001)$.

\section{Quality assessment}

One of the RCTs included was stopped prematurely due to a loss of funding. ${ }^{7}$ Nevertheless, it was considered a high quality study according to the Jadad criteria. The other two RCTs were considered to be of moderate quality because blinding was not described..$^{24}$ On funnel plot analysis, studies occupied a symmetrical distribution according to weight and converged towards the pooled effect as the weight increased (see online supplementary figure S1). Egger's regression test (see online supplementary figure S2) was also performed and showed no

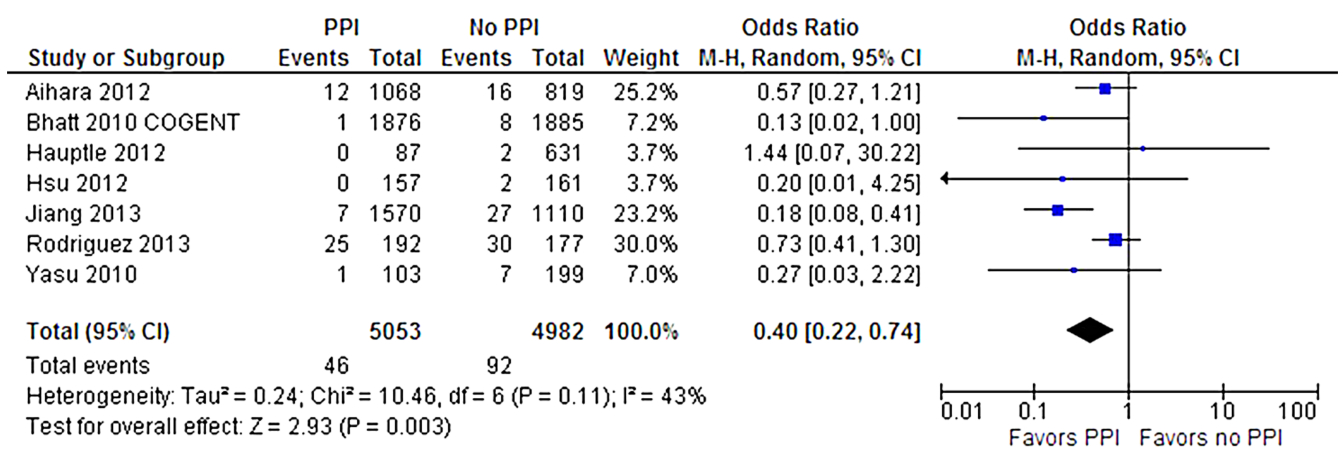

Figure 4 Forest plot of studies examining gastrointestinal bleeding between patients taking proton pump inhibitor (PPI) with clopidogrel and those taking only clopidogrel. 

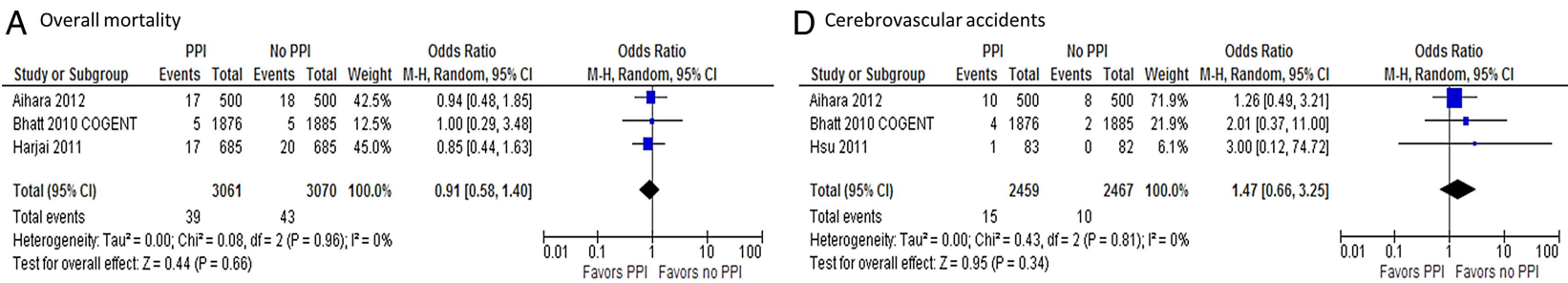

B Acute coronary syndrome

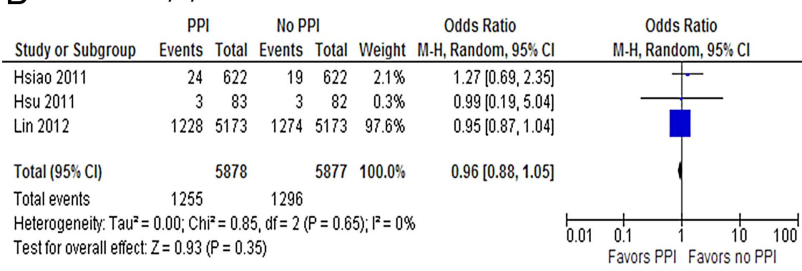

E Revascularization
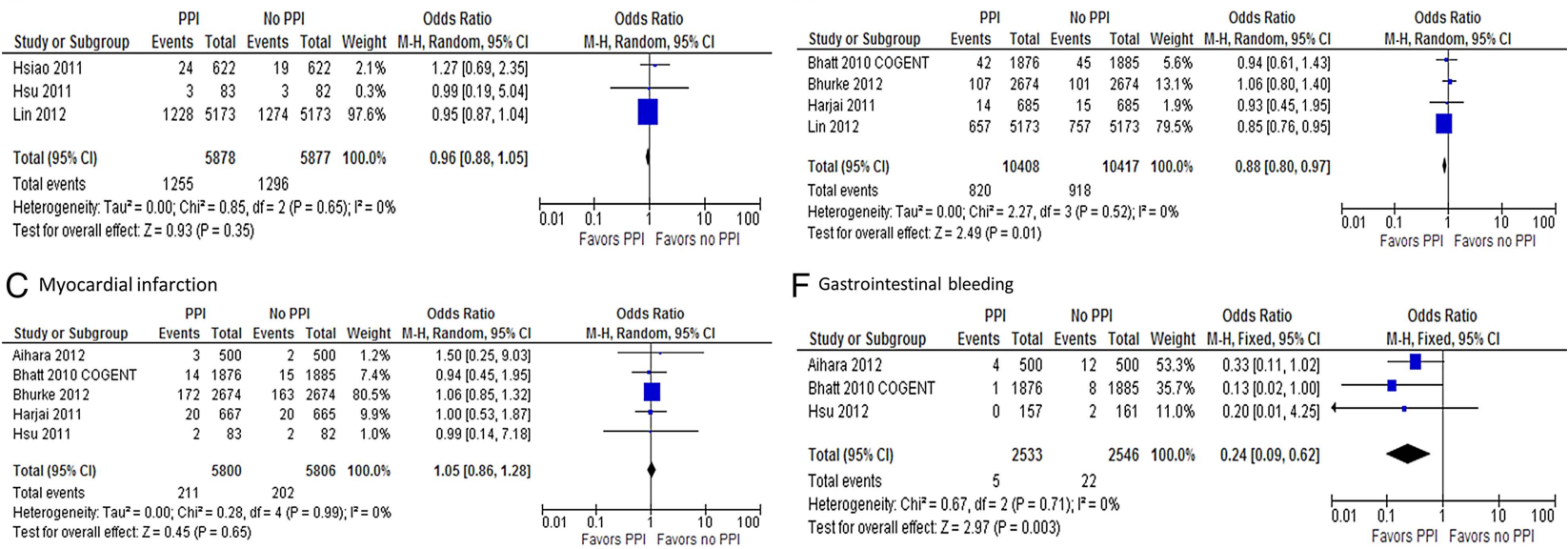

Figure 5 Forest plots of randomised controlled trials and propensity score matched studies examining outcomes between patients taking proton pump inhibitor (PPIs) with clopidogrel and those taking only clopidogrel: (A) overall mortality; (B) acute coronary syndromes; (C) myocardial infarction; (D) need for revascularisation; (E) cerebrovascular accidents; (F) gastrointestinal bleeding.

evidence of significant publication bias $(\mathrm{p}=0.48$ and 0.76 for overall mortality and MI, respectively).

\section{DISCUSSION}

The pooled analysis of all included studies included 214851 patients and found that patients who took a PPI in addition to clopidogrel had the worst outcomes, including higher overall mortality, MI, ACS, CVA, stent thrombosis and the need for revascularisation procedures. These results are consistent with previous studies and meta-analyses. ${ }^{26} 485167$ However, these data emerge mostly from nonrandomised observational studies, which are prone to selection bias and non-comparability between groups at baseline. Therefore, we conducted a separate analysis including data only from RCTs and PSM patients. In a population of 23552 patients from eight studies, we found that all ischaemic end points evaluated were not increased in the clopidogrel-PPI group (figure 5). This analysis of RCTs and PSM patients highly suggests that PPIs are a marker of increased risk, rather than a direct cause of worse outcomes.

The contrast in outcomes between unadjusted and adjusted/randomised studies is supported by findings of increased CV risk among patients taking PPI regardless of simultaneous clopidogrel use. In a population of 31704 patients who were not receiving clopidogrel, Charlot $e t a l^{73}$ found that, compared with non-PPI users, patients on PPI had an increased risk of all-cause mortality (HR 1.58; $\mathrm{p}<0.01$ ), CV mortality (HR 1.49; $\mathrm{p}<0.01$ ), MI (HR 1.13; $\mathrm{p}=0.02$ ) and CVA (HR 1.32; $\mathrm{p}<0.01)$. Furthermore, the magnitude of increased $\mathrm{CV}$ risk in the PPI group was similar between clopidogrel users and patients not receiving clopidogrel. An increased risk of ischaemic outcomes among patients taking a PPI has also been reported in concomitant use of placebo and ticagrelor. ${ }^{68} 74$

The mechanism of increased $\mathrm{CV}$ risk in patients receiving a PPI is most likely related to the difference in baseline characteristics between users and non-users of clopidogrel. In the study by Charlot et al, ${ }^{73}$ patients who received a PPI were on average 3 years older than the comparison group and also had a higher prevalence of diabetes with complications, chronic kidney injury and cerebrovascular disease at baseline. In Bhurke et al, ${ }^{30}$ patients taking clopidogrel had a higher Charlson comorbidity index at baseline, as well as a higher prevalence of heart failure. Similarly, the majority of unadjusted studies that reported an increased risk of CV events in PPI users had an unbalanced distribution of baseline characteristics, with sicker patients in the PPI group. ${ }^{31} 526367$

Our study found a decreased incidence of GI bleeding among patients taking PPIs, a result that was confirmed in patients with similar baseline characteristics (RCT/ PSM populations; figure 5F). Two different mechanisms may contribute as follows to the decreased incidence of GI bleeding with PPI use. The first is by direct inhibition 
Table 2 Subgroup analyses

\begin{tabular}{|c|c|c|c|c|}
\hline $\begin{array}{l}\text { Subgroup } \\
\text { analysis }\end{array}$ & ACS & DAPT & E/O/L & P/R \\
\hline Number of patients & $\begin{array}{l}\text { PPI: } 37015 \\
\text { No PPI: } 77060\end{array}$ & $\begin{array}{l}\text { PPI: } 38244 \\
\text { No PPI: } 40604\end{array}$ & $\begin{array}{l}\text { PPI: } 23437 \\
\text { No PPI: } 33000\end{array}$ & $\begin{array}{l}\text { PPI: } 3008 \\
\text { No PPI: } 28772\end{array}$ \\
\hline $\begin{array}{l}\text { Studies included } \\
\text { Outcomes }\end{array}$ & 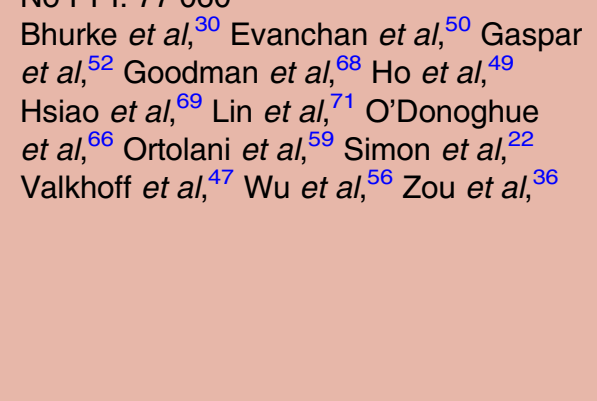 & 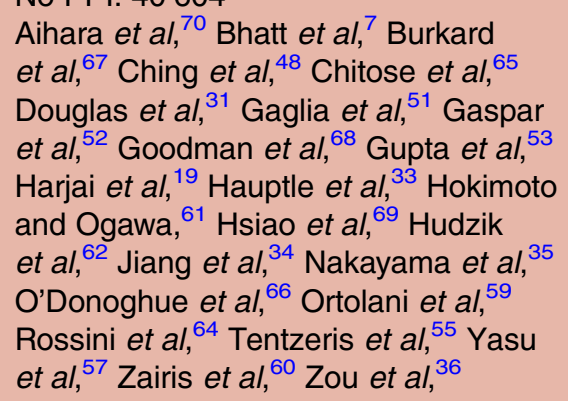 & $\begin{array}{l}\text { Bhatt et al, }{ }^{7} \text { Douglas et al, }{ }^{31} \\
\text { Gaglia et al, }{ }^{51} \text { Harjai et al, }{ }^{19} \mathrm{Hsu} \\
\text { et al, }{ }^{24} \mathrm{Hsu},{ }^{72} \text { Hudzik et } a l,{ }^{62} \\
\text { Jiang et al, },^{34} \text { Rossini } \text { et al }{ }^{64} \\
\text { Valkhoff et al, }{ }^{47} \text { Zairis et al, }{ }^{60}\end{array}$ & $\begin{array}{l}\text { Douglas et al, }{ }^{31} \text { Gaglia } \\
\text { et al, }{ }^{51} \text { Hokimoto and } \\
\text { Ogawa, }{ }^{61} \text { Rossini et } a{ }^{64} \\
\text { Valkhoff et al, }{ }^{47} \text { Yasu } \\
\text { et al, }{ }^{57}\end{array}$ \\
\hline All-cause mortality & OR $1.14 ; \mathrm{Cl} 0.94$ to $1.39 ; \mathrm{p}=0.19$ & OR $1.32 ; \mathrm{Cl} 1.10$ to $1.58 ; p=0.003$ & OR 1.23; $\mathrm{Cl} 0.72$ to $2.10 ; \mathrm{p}=0.46$ & $\begin{array}{l}\text { OR 2.01; } \mathrm{Cl} 1.20 \text { to } 3.35 ; \\
p=0.008\end{array}$ \\
\hline CV mortality & OR $0.99 ; \mathrm{Cl} 0.70$ to $1.39 ; \mathrm{p}=0.95$ & OR $1.16 ; \mathrm{Cl} 0.95$ to $1.42 ; \mathrm{p}=0.14$ & OR $1.28 ; \mathrm{Cl} 1.14$ to $1.43 ; \mathrm{p}<0.001$ & $\begin{array}{l}\text { OR } 1.96 ; \mathrm{Cl} 0.68 \text { to } 5.64 ; \\
p=0.21\end{array}$ \\
\hline ACS & OR $1.91 ; \mathrm{Cl} 0.89$ to $4.06 ; \mathrm{p}=0.09$ & OR $2.37 ; \mathrm{Cl} 1.36$ to $4.13 ; \mathrm{p}=0.002$ & NA & NA \\
\hline $\mathrm{Ml}$ & OR $1.41 ; \mathrm{Cl} 1.08$ to $1.85 ; \mathrm{p}=0.01$ & OR $1.25 ; \mathrm{Cl} 1.07$ to $1.45 ; p=0.005$ & OR $1.25 ; \mathrm{Cl} 1.09$ to $1.44 ; p=0.002$ & $\begin{array}{l}\text { OR 2.13; Cl } 1.60 \text { to } 2.85 ; \\
p<0.001\end{array}$ \\
\hline $\begin{array}{l}\text { ST (possible/ } \\
\text { probable/definite) }\end{array}$ & NA & OR $1.36 ; \mathrm{Cl} 1.10$ to $1.68 ; p=0.005$ & OR $1.08 ; \mathrm{Cl} 0.67$ to $1.73 ; \mathrm{p}=0.76$ & $\begin{array}{l}\text { OR 2.28; } \mathrm{Cl} 0.66 \text { to } 7.89 ; \\
\mathrm{p}=0.20\end{array}$ \\
\hline Revascularisation & OR $1.38 ; \mathrm{Cl} 0.82$ to $2.30 ; \mathrm{p}=0.22$ & OR $1.30 ; \mathrm{Cl} 1.08$ to $1.58 ; \mathrm{p}=0.006$ & OR $1.06 ; \mathrm{Cl} 0.73$ to $1.54 ; \mathrm{p}=0.74$ & NA \\
\hline CVA & NA & OR $1.75 ; \mathrm{Cl} 0.98$ to $3.16 ; \mathrm{p}=0.06$ & OR $2.24 ; \mathrm{Cl} 0.62$ to $8.11 ; \mathrm{p}=0.22$ & NA \\
\hline Gl bleed & NA & OR $0.31 ; \mathrm{Cl} 0.15$ to $0.65 ; \mathrm{p}=0.002$ & OR $0.17 ; \mathrm{Cl} 0.08$ to $0.36 ; p<0.001$ & NA \\
\hline
\end{tabular}


of proton pumps with subsequent suppression of acid production, which has been shown to (1) prevent stress-ulcer related bleeding in critically ill patients; ${ }^{75}$ (2) decrease rebleeding in patients with a history of ulcerrelated bleeding; ${ }^{6}$ and (3) decrease GI bleeding among patients on anticoagulants and dual anti-platelet therapy. ${ }^{76}$ Alternatively, the benefit in GI bleeding may be related to a PPI-mediated reduction in the antiplatelet effect of clopidogrel. Several pharmacokinetic studies have demonstrated a lower inhibition of platelet aggregation among patients taking a PPI in addition to clopidogrel, as compared to non-PPI clopidogrel users. ${ }^{14} 1566$ Although our findings suggest that this mechanism is not clinically relevant in terms of adverse CV outcomes, platelet aggregation plays an important role in angiogenesis and the healing of peptic ulcers; ${ }^{77}$ therefore, a lesser degree of platelet inhibition certainly has the potential to decrease GI bleeding.

As illustrated in table 2, among patient with ACS, there was no increased risk of ischaemic $\mathrm{CV}$ end points with PPI use. Patients with ACS most likely have more comorbidities and a worse prognosis at baseline compared with elective patients, which can mitigate the differences in outcomes between PPI and non-PPI users. Inhibition of the CYP450 2C19 enzyme is heterogeneous within the class of PPIs. Omeprazole, esomeprazole and lansoprazole have been shown to be the strongest inhibitors, ${ }^{11} 161720$ whereas some studies have suggested that pantoprazole and rabeprazole have no effect on the CYP450 2C19 enzyme. ${ }^{16} 20{ }^{38}$ Our meta-analysis has demonstrated that the association between adverse outcomes and concomitant PPI-clopidogrel use persists in patients taking the low-risk PPIs rabeprazole or pantoprazole. Given that these medications are not expected to have a significant interaction with clopidogrel, this finding further supports the hypothesis that use of a PPI is not the cause of increased adverse outcomes, but rather a marker of increased baseline risk.

This study has limitations. Definitions of outcomes were not reported in a substantial part of the studies, which raises the concern for reporting bias. In addition, 36 of the 39 included studies were non-randomised and are inherently more susceptible to bias. The correction of possible baseline differences between groups led to a subanalysis of randomised and PSM studies; however, this analysis included only eight studies, which did not report on all the studied outcomes. Moreover, the absence of patient-level data, common in meta-analysis designs, prevented more detailed subgroup analyses, such as interaction between different generations of drug-eluting stents and the exact role of baseline characteristics on the clopidogrel-PPI interaction. Also, this systematic review was not registered prospectively, which would have allowed feedback about the protocol, further limiting the possibility of bias. Nevertheless, we believe we have conducted a transparent and reproducible protocol. Finally, given the high number of studies included and the differences in methods and outcome definitions among them, a substantial amount of heterogeneity was encountered. This has already been observed in previous meta-analyses, and therefore only a random-effects model was used. A prespecified definition of GI bleeding and stent thrombosis was also applied to minimise bias resulting from different outcome definitions.

\section{CONCLUSION}

In summary, the results of our meta-analyses suggest that the highly controversial interaction between PPIs and clopidogrel observed in platelet aggregation studies has no clinical significance. Rather, patients who are prescribed PPIs have a higher burden of comorbidities and thus most likely have an increased risk for adverse CV events. Importantly, PPIs have the potential to significantly reduce GI bleeding among patients taking clopidogrel.

\section{Author affiliations}

${ }^{1}$ Department of Internal Medicine, University of Miami/Jackson Memorial Hospital, Miami, Florida, USA

${ }^{2}$ Department of Cardiology, Ochsner Medical Center, New Orleans, Louisiana, USA

${ }^{3}$ Saint Luke's Mid America Heart Institute, Kansas City, Missouri, USA

${ }^{4}$ Department of Cardiology, Baylor College of Medicine and Michael E. DeBakey VA Medical Center, Houston, Texas, USA

${ }^{5}$ St.Luke's Roosevelt Medical Center, New York, New York, USA

${ }^{6}$ Nephrology Department, Mount Sinai Hospital, New York, New York, USA

${ }^{7}$ Department of Cardiology, University of Turin, Turin, Italy

Contributors AMB, JPR were involved in the conception and design of the study; analysis and interpretation of data and drafting of the manuscript; final approval of the manuscript submitted. RNC was involved in the conception and design of the study; data collection and drafting of the manuscript; final approval of the manuscript submitted. JJD, GNN were involved in the analysis and interpretation of data; critical revision of the manuscript for important intellectual content; final approval of the manuscript submitted. DCG, FYBM, $\mathrm{GE}-\mathrm{H}$ were involved in the data collection; final approval of the manuscript submitted. IK was involved in the analysis and interpretation of data; final approval of the manuscript submitted. SG, MI were involved in the statistical analysis; final approval of the manuscript submitted.

Competing interests None declared.

Provenance and peer review Not commissioned; externally peer reviewed.

Data sharing statement No additional data are available.

Open Access This is an Open Access article distributed in accordance with the Creative Commons Attribution Non Commercial (CC BY-NC 4.0) license, which permits others to distribute, remix, adapt, build upon this work noncommercially, and license their derivative works on different terms, provided the original work is properly cited and the use is non-commercial. See: http:// creativecommons.org/licenses/by-nc/4.0/

\section{REFERENCES}

1. Levine GN, Bates ER, Blankenship JC, et al. 2011 ACCF/AHA/SCA Guideline for Percutaneous Coronary Intervention: a report of the American College of Cardiology Foundation/American Heart Association Task Force on Practice Guidelines and the Society for Cardiovascular Angiography and Interventions. Circulation 2011;124:e574-651.

2. O'Gara PT, Kushner FG, Ascheim DD, et al. 2013 ACCF/AHA guideline for the management of ST-elevation myocardial infarction: a report of the American College of Cardiology Foundation/American Heart Association Task Force on Practice Guidelines. Circulation 2013;127:e362-425. 
3. Writing Committee MembersJneid H, Anderson JL, Wright RS, et al., American College of Cardiology Foundation; American Heart Association Task Force on Practice Guidelines. 2012 ACCF/AHA focused update of the guideline for the management of patients with unstable angina/Non-ST-elevation myocardial infarction (updating the 2007 guideline and replacing the 2011 focused update): a report of the American College of Cardiology Foundation/American Heart Association Task Force on practice guidelines. Circulation 2012;126:875-910.

4. Yusuf S, Zhao F, Mehta SR, et al. Clopidogrel in unstable angina to prevent recurrent events trial I: effects of clopidogrel in addition to aspirin in patients with acute coronary syndromes without ST-segment elevation. N Engl J Med 2001;345:494-502.

5. Steinhubl SR, Berger PB, Mann JT III, et al., CREDO Investigators. Clopidogrel for the Reduction of Events During Observation. Observation CICftRoED: early and sustained dual oral antiplatelet therapy following percutaneous coronary intervention: a randomized controlled trial. JAMA 2002;288:2411-20.

6. Lai KC, Lam SK, Chu KM, et al. Lansoprazole for the prevention of recurrences of ulcer complications from long-term low-dose aspirin use. N Engl J Med 2002;346:2033-8.

7. Bhatt DL, Cryer BL, Contant CF, et al. Clopidogrel with or without omeprazole in coronary artery disease. $N$ Engl $\mathrm{J} \mathrm{Med}$ 2010;363:1909-17.

8. Siller-Matula JM, Jilma B, Schror K, et al. Effect of proton pump inhibitors on clinical outcome in patients treated with clopidogrel: a systematic review and meta-analysis. $J$ Thromb Haemost 2010;8:2624-41.

9. Hulot JS, Bura A, Villard E, et al. Cytochrome P450 2C19 loss-of-function polymorphism is a major determinant of clopidogrel responsiveness in healthy subjects. Blood 2006;108:2244-7.

10. Scott SA, Sangkuhl K, Stein CM, et al. Clinical Pharmacogenetics Implementation Consortium guidelines for CYP2C19 genotype and clopidogrel therapy: 2013 update. Clin Pharmacol Ther 2013;94:317-23.

11. Li XQ, Andersson TB, Ahlstrom M, et al. Comparison of inhibitory effects of the proton pump-inhibiting drugs omeprazole, esomeprazole, lansoprazole, pantoprazole, and rabeprazole on human cytochrome P450 activities. Drug Metab Dispos 2004;32:821-7.

12. Ishizaki T, Horai $Y$. Review article: cytochrome P450 and the metabolism of proton pump inhibitors-emphasis on rabeprazole. Aliment Pharmacol Ther 1999;13(Suppl 3):27-36.

13. Zuern CS, Geisler T, Lutilsky N, et al. Effect of comedication with proton pump inhibitors (PPIs) on post-interventional residual platelet aggregation in patients undergoing coronary stenting treated by dual antiplatelet therapy. Thromb Res 2010;125:e51-54.

14. Yun $\mathrm{KH}$, Rhee SJ, Park HY, et al. Effects of omeprazole on the antiplatelet activity of clopidogrel. Int Heart J 2010;51:13-16.

15. Gilard M, Arnaud B, Cornily JC, et al. Influence of omeprazole on the antiplatelet action of clopidogrel associated with aspirin: the randomized, double-blind OCLA (Omeprazole CLopidogrel Aspirin) study. J Am Coll Cardiol 2008;51:256-60.

16. Angiolillo DJ, Gibson CM, Cheng S, et al. Differential effects of omeprazole and pantoprazole on the pharmacodynamics and pharmacokinetics of clopidogrel in healthy subjects: randomized, placebo-controlled, crossover comparison studies. Clin Pharmacol Ther 2011;89:65-74.

17. US-Food-and-Drug-Adminstration. Update to the labeling of Clopidogrel Bisulfate (marketed as Plavix) to alert healthcare professionals about a drug interaction with omeprazole (marketed as Prilosec and Prilosec OTC). http://www.fda.gov/ Drugs/DrugSafety/ PostmarketDrug-SafetylnformationforPatientsandProviders/Drug SafetyInformationforHeathcareProfessionals/-ucm190787.htm2009 (accessed 28 May 2014).

18. Abraham NS, Hlatky MA, Antman EM, et al. ACCF/ACG/AHA 2010 expert consensus document on the concomitant use of proton pump inhibitors and thienopyridines: a focused update of the ACCF/ACG/AHA 2008 expert consensus document on reducing the gastrointestinal risks of antiplatelet therapy and NSAID use. A Report of the American College of Cardiology Foundation Task Force on Expert Consensus Documents. J Am Coll Cardiol 2010;56:2051-66.

19. Harjai KJ, Shenoy C, Orshaw P, et al. Clinical outcomes in patients with the concomitant use of clopidogrel and proton pump inhibitors after percutaneous coronary intervention: an analysis from the Guthrie Health Off-Label Stent (GHOST) investigators. Circ Cardiovasc Interv 2011;4:162-70.

20. Juurlink DN, Gomes T, Ko DT, et al. A population-based study of the drug interaction between proton pump inhibitors and clopidogrel. CMAJ 2009;180:713-18.

21. Schmidt M, Johansen MB, Robertson DJ, et al. Concomitant use of clopidogrel and proton pump inhibitors is not associated with major adverse cardiovascular events following coronary stent implantation. Aliment Pharmacol Ther 2012;35:165-74.

22. Simon T, Steg PG, Gilard M, et al. Clinical events as a function of proton pump inhibitor use, clopidogrel use, and cytochrome P450 2C19 genotype in a large nationwide cohort of acute myocardial infarction: results from the French Registry of Acute ST-Elevation and Non-ST-Elevation Myocardial Infarction (FAST-MI) registry. Circulation 2011;123:474-82

23. van Boxel OS, van Oijen MG, Hagenaars MP, et al. Cardiovascular and gastrointestinal outcomes in clopidogrel users on proton pump inhibitors: results of a large Dutch cohort study. Am J Gastroenterol 2010;105:2430-6; quiz 2437.

24. Hsu PI, Lai KH, Liu CP. Esomeprazole with clopidogrel reduces peptic ulcer recurrence, compared with clopidogrel alone, in patients with atherosclerosis. Gastroenterology 2011;140:791-8.

25. Kwok CS, Loke YK. Meta-analysis: the effects of proton pump inhibitors on cardiovascular events and mortality in patients receiving clopidogrel. Aliment Pharmacol Ther 2010;31:810-23.

26. Gerson LB, McMahon D, Olkin I, et al. Lack of significant interactions between clopidogrel and proton pump inhibitor therapy: meta-analysis of existing literature. Dig Dis Sci 2012;57:1304-13.

27. Kwok CS, Jeevanantham V, Dawn B, et al. No consistent evidence of differential cardiovascular risk amongst proton-pump inhibitors when used with clopidogrel: meta-analysis. Int $J$ Cardiol 2013;167:965-74.

28. Huang B, Huang Y, Li Y, et al. Adverse cardiovascular effects of concomitant use of proton pump inhibitors and clopidogrel in patients with coronary artery disease: a systematic review and meta-analysis. Arch Med Res 2012;43:212-24.

29. Focks JJ, Brouwer MA, van Oijen MG, et al. Concomitant use of clopidogrel and proton pump inhibitors: impact on platelet function and clinical outcome- a systematic review. Heart 2013;99:520-7.

30. Bhurke SM, Martin BC, Li C, et al. Effect of the clopidogrel-proton pump inhibitor drug interaction on adverse cardiovascular events in patients with acute coronary syndrome. Pharmacotherapy 2012;32:809-18.

31. Douglas IJ, Evans SJ, Hingorani AD, et al. Clopidogrel and interaction with proton pump inhibitors: comparison between cohort and within person study designs. BMJ 2012;345:e4388.

32. Garcia Rodriguez LA, Johansson S, Cea Soriano L. Use of clopidogrel and proton pump inhibitors after a serious acute coronary event: risk of coronary events and peptic ulcer bleeding. Thromb Haemost 2013;110:1014-24.

33. Hauptle R, Weilenmann D, Schneider T, et al. Individualised PPI prescription in patients on combination antiplatelet therapy and upper gastrointestinal events after percutaneous coronary intervention: a cohort study. Wien Med Wochenschr2012;162:67-73.

34. Jiang Z, Wu H, Duan Z, et al. Proton-pump inhibitors can decrease gastrointestinal bleeding after percutaneous coronary intervention. Clin Res Hepatol Gastroenterol 2013;37:636-41.

35. Nakayama A, Morita $\mathrm{H}$, Ando J, et al. Adverse cardiovascular outcomes associated with concurrent use of clopidogrel or ticlopidine and proton-pump inhibitors in patients undergoing percutaneous coronary intervention. Heart Vessels 2013;28:292-300.

36. Zou JJ, Chen SL, Tan J, et al. Increased risk for developing major adverse cardiovascular events in stented Chinese patients treated with dual antiplatelet therapy after concomitant use of the proton pump inhibitor. PLOS ONE 2014;9:e84985.

37. Mauri L, Hsieh WH, Massaro JM, et al. Stent thrombosis in randomized clinical trials of drug-eluting stents. $N$ Engl $J$ Med 2007;356:1020-9.

38. Hokimoto S, Mizobe M, Akasaka T, et al. Impact of CYP2C19 polymorphism and proton pump inhibitors on platelet reactivity to clopidogrel and clinical outcomes following stent implantation. Thromb Res 2014;133:599-605.

39. eWells GA, Shea B, O'Connell D, et al. The Newcastle-Ottawa Scale (NOS) for assessing the quality of nonrandomized studies in meta-analyses. http://www.ohri.ca/ programs/-clinical-epidemiology/ oxford.asp. (accessed 13 Mar 2014).

40. Shabanzadeh DM, Sorensen LT. Laparoscopic surgery compared with open surgery decreases surgical site infection in obese patients: a systematic review and meta-analysis. Ann Surg 2012;256:934-45

41. Lanitis S, Tekkis PP, Sgourakis G, et al. Comparison of skin-sparing mastectomy versus non-skin-sparing mastectomy for breast cancer: a meta-analysis of observational studies. Ann Surg 2010;251:632-9.

42. Jadad AR, Moore RA, Carroll D, et al. Assessing the quality of reports of randomized clinical trials: is blinding necessary? Control Clin Trials 1996;17:1-12.

43. Duval S, Tweedie R. Trim and fill. Biometrics 2000;56:455-63. 
44. Moher D, Liberati A, Tetzlaff J, et al. Preferred reporting items for systematic reviews and meta-analyses: the PRISMA statement. BMJ 2009;339:b2535.

45. Higgins JP, Thompson SG, Deeks JJ, et al. Measuring inconsistency in meta-analyses. BMJ 2003;327:557-60.

46. Juurlink DN, Gomes T, Mamdani MM, et al. The safety of proton pump inhibitors and clopidogrel in patients after stroke. Stroke 2011;42:128-32.

47. Valkhoff VE, $t$ Jong GW, Van Soest EM, et al. Risk of recurrent myocardial infarction with the concomitant use of clopidogrel and proton pump inhibitors. Aliment Pharmacol Ther 2011;33:77-88.

48. Ching GG, Li D, Baker WL, et al. Major adverse cardiac events among postpercutaneous coronary intervention patients on clopidogrel and proton pump inhibitors. Conn Med 2012;76:205-11.

49. Ho PM, Maddox TM, Wang L, et al. Risk of adverse outcomes associated with concomitant use of clopidogrel and proton pump inhibitors following acute coronary syndrome. JAMA 2009;301:937-44.

50. Evanchan J, Donnally MR, Binkley P, et al. Recurrence of acute myocardial infarction in patients discharged on clopidogrel and a proton pump inhibitor after stent placement for acute myocardial infarction. Clin Cardiol 2010;33:168-71.

51. Gaglia MA Jr, Torguson R, Hanna N, et al. Relation of proton pump inhibitor use after percutaneous coronary intervention with drug-eluting stents to outcomes. Am J Cardiol 2010;105:833-8.

52. Gaspar A, Ribeiro S, Nabais S, et al. Proton pump inhibitors in patients treated with aspirin and clopidogrel after acute coronary syndrome. Rev Port Cardiol 2010;29:1511-20.

53. Gupta E, Bansal D, Sotos J, et al. Risk of adverse clinical outcomes with concomitant use of clopidogrel and proton pump inhibitors following percutaneous coronary intervention. Dig Dis Sci 2010:55:1964-8.

54. Kreutz RP, Stanek EJ, Aubert R, et al. Impact of proton pump inhibitors on the effectiveness of clopidogrel after coronary stent placement: the clopidogrel Medco outcomes study. Pharmacotherapy 2010;30:787-96.

55. Tentzeris I, Jarai R, Farhan S, et al. Impact of concomitant treatment with proton pump inhibitors and clopidogrel on clinical outcome in patients after coronary stent implantation. Thromb Haemost 2010;104:1211-18

56. Wu CY, Chan FK, Wu MS, et al. Histamine2-receptor antagonists are an alternative to proton pump inhibitor in patients receiving clopidogrel. Gastroenterology 2010:139:1165-71.

57. Yasu T, Ikee R, Miyasaka Y, et al. [Efficacy and safety of concomitant use of rabeprazole during dual-antiplatelet therapy with clopidogrel and aspirin after drug-eluting stent implantation: a retrospective cohort study]. Yakugaku zasshi 2010;130:1743-50.

58. Munoz-Torrero JF, Escudero D, Suarez C, et al. Factores de Riesgo y EAl: concomitant use of proton pump inhibitors and clopidogrel in patients with coronary, cerebrovascular, or peripheral artery disease in the factores de Riesgo y ENfermedad Arterial (FRENA) registry. J Cardiovasc Pharmacol 2011;57:13-19.

59. Ortolani $P$, Marino M, Marzocchi A, et al. One-year clinical outcome in patients with acute coronary syndrome treated with concomitant use of clopidogrel and proton pump inhibitors: results from a regional cohort study. J Cardiovasc Med (Hagerstown) 2012;13:783-9.

60. Zairis MN, Tsiaousis GZ, Patsourakos NG, et al. The impact of treatment with omeprazole on the effectiveness of clopidogrel drug therapy during the first year after successful coronary stenting. Can $J$ Cardiol 2010;26:e54-57.

61. Hokimoto $S$, Ogawa $H$. Is it safe to use a proton pump inhibitor with clopidogrel? A comparison of clopidogrel with and without rabeprazole in Japan. Digestive Disease Week New Orleans, United States. 2010;138:S498.
62. Hudzik B, Szkodzinski J, Danikiewicz A, et al. Effect of omeprazole on the concentration of interleukin- 6 and transforming growth factor-beta1 in patients receiving dual antiplatelet therapy after percutaneous coronary intervention. Eur Cytokine Netw 2010;21:257-63.

63. Banerjee S, Weideman RA, Weideman MW, et al. Effect of concomitant use of clopidogrel and proton pump inhibitors after percutaneous coronary intervention. Am J Cardiol 2011;107:871-8.

64. Rossini R, Capodanno D, Musumeci G, et al. Safety of clopidogrel and proton pump inhibitors in patients undergoing drug-eluting stent implantation. Coron Artery Dis 2011;22:199-205.

65. Chitose T, Hokimoto S, Oshima S, et al., Kumamoto Intervention Conference Study (KICS) Investigators. Clinical outcomes following coronary stenting in Japanese patients treated with and without proton pump inhibitor. Circ J 2012;76:71-8.

66. O'Donoghue ML, Braunwald E, Antman EM, et al Pharmacodynamic effect and clinical efficacy of clopidogrel and prasugrel with or without a proton-pump inhibitor: an analysis of two randomised trials. Lancet 2009;374:989-97.

67. Burkard T, Kaiser CA, Brunner-La Rocca H, et al. Combined clopidogrel and proton pump inhibitor therapy is associated with higher cardiovascular event rates after percutaneous coronary intervention: a report from the BASKET trial. $J$ Intern Med 2012;271:257-63.

68. Goodman SG, Clare R, Pieper KS, et al. Association of proton pump inhibitor use on cardiovascular outcomes with clopidogrel and ticagrelor: insights from the platelet inhibition and patient outcomes trial. Circulation 2012:125:978-86.

69. Hsiao FY, Mullins CD, Wen YW, et al. Relationship between cardiovascular outcomes and proton pump inhibitor use in patients receiving dual antiplatelet therapy after acute coronary syndrome. Pharmacoepidemiol Drug Saf 2011;20:1043-9.

70. Aihara $\mathrm{H}$, Sato A, Takeyasu N, et al. Effect of individual proton pump inhibitors on cardiovascular events in patients treated with clopidogrel following coronary stenting: results from the Ibaraki Cardiac Assessment Study Registry. Catheter Cardiovasc Interv 2012;80:556-63.

71. Lin CF, Shen LJ, Wu FL, et al. Cardiovascular outcomes associated with concomitant use of clopidogrel and proton pump inhibitors in patients with acute coronary syndrome in Taiwan. $\mathrm{Br} J$ Clin Pharmacol 2012;74:824-34.

72. Hsu P. Proton pump inhibitor prevents clinically significant upper gastrointestinal events in clopidogrel users with ulcer history. Digestive Disease Week San Diego, United States. 2012;142:S27.

73. Charlot M, Ahlehoff O, Norgaard ML, et al. Proton-pump inhibitors are associated with increased cardiovascular risk independent of clopidogrel use: a nationwide cohort study. Ann Intern Med 2010;153:378-86.

74. Dunn SP, Steinhubl SR, Bauer D, et al. Impact of proton pump inhibitor therapy on the efficacy of clopidogrel in the CAPRIE and CREDO trials. J Am Heart Assoc 2013;2:e004564.

75. Stollman N, Metz DC. Pathophysiology and prophylaxis of stress ulcer in intensive care unit patients. J Crit Care 2005;20:35-45.

76. $\mathrm{Ng} \mathrm{FH}$, Wong SY, Lam KF, et al. Gastrointestinal bleeding in patients receiving a combination of aspirin, clopidogrel, and enoxaparin in acute coronary syndrome. Am J Gastroenterol 2008;103:865-71.

77. Bhatt DL, Scheiman J, Abraham NS, et al. ACCF/ACG/AHA 2008 expert consensus document on reducing the gastrointestinal risks of antiplatelet therapy and NSAID use: a report of the American College of Cardiology Foundation Task Force on Clinical Expert Consensus Documents. J Am Coll Cardiol 2008;52:1502-17. 